\title{
DECISIONES AL FINAL DE LA VIDA: CASO ASOCIACIÓN HOGAR DE ANCIANOS DE COTO BRUS
}

\section{DECISIONS AT THE END OF LIFE: CASE OF THE NURSING HOME ASSOCIATION OF COTO BRUS}

\author{
Roxana Hernández Vargas \\ Universidad de Costa Rica \\ roxana.hernandezvargas@gmail.com
}

\author{
Randall Jiménez Retana \\ Universidad de Costa Rica \\ ranjim77@gmail.com
}

\section{Georgina Morera Quesada \\ Universidad de Costa Rica \\ geomoreraq@gmail.com}

Recibido: 23 de setiembre / Aceptado: 4 de octubre / Publicado: 15 de octubre, 2020

\begin{abstract}
Resumen
El presente artículo se propone exponer las percepciones entorno a las decisiones en el proceso del final de la vida de las personas residentes en Hogar de Ancianos de Coto Brus. Es un estudio de tipo cualitativo, descriptivo y transversal, en el cual se entrevistaron 23 residentes. Para la recolección de datos, se elaboró un cuestionario estructurado con 13 preguntas de múltiple opción. La organización y la presentación de los datos, en forma de estadística descriptiva, se realizó mediante el software Microsoft Excel. Para la presentación, se utilizó como herramienta Canva. Como parte de las conclusiones, se constató la importancia para los sujetos el expresar las expectativas respecto de las voluntades anticipadas. Asimismo, se discute la necesidad de considerar la donación de órganos y la relación familiar como elementos que requieren explorarse más, debido a la carga que supone para las personas participantes.
\end{abstract}

Palabras clave:

Final de la vida; envejecimiento; enfermedad; autonomía; vulnerabilidad; toma de decisiones. 


\begin{abstract}
The purpose of this text is to present the perceptions regarding the decisions in the end-oflife process of the residents of the Coto Brus Nursing Home. It is a qualitative, descriptive and cross-sectional study, in which 23 residents were interviewed. For data collection, a structured questionnaire was developed with 13 multiple-choice questions. The organization and presentation of the data, in the form of descriptive statistics, was carried out using Microsoft Excel software and for the presentation of the data, it was used as a Canva tool. As part of the conclusions, the importance of expressing expectations regarding advance directives was verified for the subjects. Likewise, the need to consider organ donation was discussed, as well as the family relationship as elements that require further exploration, due to the burden it entails for the participants.
\end{abstract}

\title{
Keywords:
}

End of life, aging, illness, autonomy, vulnerability, decision making.

\section{Introducción}

La propuesta del presente acercamiento se inscribe en el interés por favorecer la discusión sobre los postulados necesarios para que las personas adultas mayores tomen decisiones previas a situaciones de enfermedad o accidentes graves. Así, se evitará la aplicación de medidas que no fueron consideradas por los sujetos, hombres y mujeres, en condiciones de competencia y capacidad para la autodeterminación.

La consulta y la escucha activa a los intereses, y los deseos manifestados por poblaciones que se encuentran con situaciones especiales relacionadas con su salud y edad, tienen que ser actitudes presentes en la sociedad. En este sentido, la indagación acerca de futuras decisiones, ante escenarios hipotéticos, que guardan relación con procesos de salud y edad, es una consideración mínima para procurar el respeto a la autonomía personal.

Las personas buscan establecer, en medio de las circunstancias particulares, escenarios que les procuren seguridad y estabilidad. Al respecto plantea Jiménez (2016, p. 96):

La realidad de cada persona, en el proceso del final de la vida, es compleja. Un rasgo común es que las personas no desean sufrir dolores que pueden ser paliados con los medios de los que dispone la medicina en el siglo XX. 
Por ello, la indagación sobre cuáles son las percepciones respecto de decisiones en el proceso del final de la vida requiere consultarse con mayor profundidad en las poblaciones que se encuentran en hogares y centros de cuido.

\section{Fundamento teórico}

En diversas circunstancias, una persona podría resultar imposibilitada de expresar sus voluntades o deseos ante un accidente o agravamiento de salud repentino. La muerte vedada o la enfermedad silenciada tienen su correlato en temas no explorados o discutidos por la falta de interés académico o por tabúes sociales y culturales. Elizabeth Kubler-Ross expresa lo siguiente:

...Cuando un paciente está gravemente enfermo, a menudo se le trata como una persona sin derecho a opinar. A menudo es otro quien toma la decisión de si hay que hospitalizarlo o no, cuándo y dónde. ¡Costaría tan poco recordar que la persona enferma también tiene sentimientos, deseos y opiniones, y-lo más importante de todo- tiene derecho a ser oída! (1993, p. 22)

Ahora bien, cabe preguntarse, si la razón para no consultar los deseos y las decisiones de la persona enferma radica en la falta de conciencia al tratarse de un ser con sentimientos y capacidad de decisión, o si de fondo está el temor a la respuesta, la cual podría no coincidir con la voluntad de la persona ejecutora/cuidadora/responsable, o que abre la posibilidad del diálogo sobre otros temas, realidades o sentimientos, que no se quieren reconocer o enfrentar. Aún más, queda la duda de si la mayor dificultad es elaborar la pregunta. La consulta en sí misma significa poner en palabras y llevar al intercambio, entre los sujetos involucrados, un tema y una realidad que podrían aún no haberse aceptado. Se juega, quizá, con la fantasía de que mientras no se diga y si no se consulta, se podría seguir creyendo que no existe. De ahí, surge la importancia de explicitar y defender el derecho de decidir de la persona enferma, porque ello puede ser el impulsor no solo para resguardar dicho derecho, sino también que impulse un encuentro y un diálogo que, sin duda, serán medulares en un proceso de resolución ante una situación difícil que debe ser enfrentada. 
León Tolstói ofrece una pieza literaria especial para considerar todo aquello que no debe realizarse. Se alude al libro titulado La muerte de Iván Ilitch. En este libro se lee:

...lo que más atormentaba a Iván Ilitch, además de aquella mentira o como consecuencia de ella, era que nadie le tuviese lástima como a él le habría gustado que se la tuviesen. En ciertos momentos, después de largas crisis dolorosas, por vergüenza que le diera confesárselo, habría querido por encima de todo que le tuvieran lástima como a un niño enfermo. Sentía ganas de que lo acariciasen, de que lo besasen, de que llorasen cerca de él, como se acaricia y se consuela a los niños. (1984, p. 111)

En cuanto al cariño, la cercanía y la consideración hacia los seres que se encuentran en situaciones de desventaja y vulnerabilidad, es necesario replantearlas en nuestra sociedad, que cada vez se envejece más, se requiere volver a redefinir los estatutos mediante los cuales se establecen los mecanismos para apoyar a las poblaciones en edad avanzada. Esta consideración permitirá que puedan ejercer su autonomía, a pesar de su edad, enfermedades y de los procesos propios de su estado.

Quizá, en la vida de toda persona, como también lo fue en la vida de Iván Ilitch, juega un papel fundamental la relación y las conductas afectivas acostumbradas por las personas antes de su enfermedad. Queda pensar si, en los casos en los que no existían conductas de consuelo y empatía ante las dificultades y los sufrimientos cotidianos en época de salud, podrán surgir en situaciones de enfermedad. Por lo tanto, resulta esencial reconocerlo como un elemento fundamental dentro de los procesos de acompañamiento y considerarlo como el derecho a la afectividad y a la compasión en situaciones de vulnerabilidad.

Ante los escenarios planteados en los párrafos anteriores, aflora una realidad que no es inusual en procesos de enfermedad grave o en el final de la vida. Puede suceder que el vacío, que experimenta la persona enferma, no sea llenado por sus personas cercanas, sino que quien cumpla ese papel sean las personas operarias de la salud o aquellas que se interesan en apoyar el proceso, pero que no forman parte del núcleo familiar. 
Este tipo de consultas, a los posibles sujetos afectados, favorece que las personas operarias en salud mantengan más confianza respecto de los deseos y las preferencias de los usuarios en materia de valores, visiones de mundo y de vida. Además, les permite identificar los límites racionales de sus acciones y recomendaciones ante las circunstancias apremiantes, que una enfermedad o accidente incapacitante puedan generar. Ante estas situaciones o la aparición de una enfermedad degenerativa o terminal, el paciente entabla un diálogo acerca de sus valores, de lo que juzga como calidad de vida, encarnizamiento terapéutico, como tratamientos fútiles, entre otras nociones relevantes y relacionadas con el proceso del final de la vida.

\section{Proceso del final de la vida}

Intrínsecamente, los sujetos se encuentran en una situación de vulnerabilidad, debido tanto a aspectos del ámbito de lo físico, lo biológico, lo social o lo político poseen circunstancias que los colocan en tensión, que pueden provocar disminución de sus habilidades y sus capacidades. Este dato implica aceptar que la vida es imprevisible, por lo que la anticipación de estos escenarios y el cuidado de ciertos procesos naturales en el ser humano favorecen la limitación de esta incertidumbre. "La muerte se presenta en la sociedad occidental como miedo, silencio respetuoso o de sustracción del derecho" (Alcoberro, 2013, p. 183).

Cabe pensar que el acercamiento a la persona enferma impacta al otro ser al reconocer que la vida es incierta y vulnerable, pero no solamente la vida de la persona enferma, sino también la propia vida. Preguntar y darles un espacio a las decisiones de la persona vulnerada, obliga al acercamiento profundo de la vivencia, que podría no estar preparada para aceptar y menos aún para atenderla.

Sin embargo, "hay ciertas poblaciones o grupos humanos que están más expuestos a condiciones de vulnerabilidad" (Cf. Beauchamp y Childres, 2013, pp. 267-268). Dentro de estos grupos, estarían los sujetos en situación de pobreza, con enfermedades mentales, condición de vejez, discapacidad, personas incapacitadas por accidentes o con enfermedades en estado terminal.

En el proceso del final de la vida se pueden encontrar dos principios que, para el presente trabajo, se consideran trascendentales: autonomía y vulnerabilidad. Los sujetos podrían encontrarse en una condición de 
vulnerabilidad, pero que, por las facilidades que ofrece el sistema de salud o las posibilidades económicas privadas, pueden ejercer su autonomía en su proceso de enfermedad o, previsiblemente, con los documentos de voluntades anticipadas (Cf. Cabrera y Sales, 2013, pp. 255-270). Desde la filosofía platónica se enfatizaba en definir la vida como aprendizaje para la muerte (Cf. Fedón).

Dentro de este proceso está la condición de la soledad o el abandono. La muerte vedada implica que esa condición es íntima, del entorno espacial más próximo a cada sujeto. Por tanto, se sitúa a la muerte en un contexto público, institucionalizado, desarraigado del ámbito familiar. La muerte se traslada a los hospitales (Cabrera y Sales, 2013), donde, por la propia dinámica de las sociedades modernas, se asume que son los hospicios los lugares privilegiados para que traten a las personas en estado de final de la vida. La muerte es la constatación de que la técnica y la ciencia han fracasado. Esta disyuntiva puede generar un peligro: el encarnizamiento terapéutico del personal operario de la salud por sostener una vida a pesar de la propia persona y de su calidad de vida.

Aunque en Costa Rica la reflexión sobre voluntades anticipadas es incipiente (Jiménez, 2016; Jiménez, 2017), estos documentos son propicios, porque favorecen el ejercicio de la autonomía con previsión a una enfermedad terminal o un accidente incapacitante. Los mejores modelos permiten una estrategia de atención desde lo biopsicosocial-espiritual. Además, posibilita visualizar otros sitios de acción para los diferentes actores en el proceso.

En el proceso del final de la vida es recomendable tener presente en todo momento lo siguiente:

...la autonomía moral incluye el derecho de morir ... clausurando el propio tiempo vital de una manera acorde al máximo con la forma como uno ha pretendido vivir, es decir, de acuerdo con sus creencias, a sus propios deseos e incluso a sus expectativas singulares. La autonomía significa pertenecerse a sí mismo pues, en última instancia: ¿si uno no puede pertenecerse a sí mismo, a quién podría pertenecer? (Alcoberro, 2013, p.189) 
El ejercicio de la libertad y de la consideración de las decisiones personales siempre es fundamental, porque favorece la confianza en un proceso que ya es incierto y cambiante.

\section{Método}

Se trata de un estudio de tipo cualitativo, descriptivo y transversal. Se enmarca en las acciones desarrolladas durante los "Campamentos de Desarrollo Humano" en el año 2019 en la Sede del Sur, adscrita al proyecto TC-657 "El Sur Emprende", como una actividad de extensión. Para desarrollar esta iniciativa, de previo, se obtuvo el visto bueno de las personas responsables del Hogar de Ancianos de Coto Brus. Seguidamente, se inscribió la actividad como parte del proyecto de extensión mencionado. Luego, se efectuó un conversatorio con las personas residentes sobre "voluntades anticipadas", espacio para escuchar y dilucidar inquietudes respecto del tema. Posterior a dicho conversatorio, se estableció la logística con la administración del Hogar de Ancianos para realizar la entrevista. Se aplicó el instrumento durante los días lunes y en horario de la mañana para la comodidad de las personas residentes.

Después de este primer contacto con la población residente, se entrevistaron 23 personas del Hogar de Ancianos de Coto Brus, Puntarenas. La población total se conforma por 37 personas. Los criterios de inclusión fueron los siguientes: residir permanentemente en el Hogar de Ancianos de Coto Brus y que las personas aceptaran participar, luego de la lectura y la comprensión del documento de consentimiento libre e informado (DCLI), el cual fue presentado por las personas investigadoras. Los criterios de exclusión restringieron casos en los que no podían contestar debido a estar entubados, en cuidados más críticos (11 sujetos). Asimismo, aquellas personas que se encontraban fuera del hogar por visita a la familia (3 sujetos).

Los sujetos alfabetizados recibieron cuestionarios para responder por sí mismos; las preguntas fueron leídas por las personas investigadoras a quienes no sabían leer. Las respuestas fueron registradas en el instrumento de evaluación. La elección del Hogar de Ancianos de Coto Brus, se debió a la accesibilidad y los servicios que brindan a la población residente. Es un hogar de referencia en la región del Pacífico Sur. 
Para la recolección de datos, se elaboró un cuestionario estructurado con 13 preguntas de múltiple opción. Para su elaboración, se realizó una búsqueda bibliográfica en varias bases de datos: Science Direct y Clinical Key, con la frase: Advance Healthcare Planning y planificación anticipada de asistencia sanitaria. La batería de preguntas incluía datos sociodemográficos y preferencias ante situaciones del proceso del final de la vida: dónde morir, quién quisiera que lo cuidara, maniobras de resucitación, donación de órganos, alimentación artificial y disposición del cuerpo una vez acaecida la muerte. Se exploró el conocimiento sobre las decisiones al final de la vida. El cuestionario se aplicó por medio de una entrevista estructurada con las personas participantes en un ambiente reservado y adecuadamente preparado para dicho proceso.

La organización y la presentación de los datos, en forma de estadística descriptiva, se realizó mediante el software Microsoft Excel y para la presentación se utilizó como herramienta Canva.

\section{Resultados}

El Hogar de Ancianos de Coto Brus funciona como una Asociación sin fines de lucro, de interés social desde el año 1989. Se ubica en la cabecera de este cantón: San Vito (Hogar de Ancianos, 1989). Este centro se especializa en atención a la persona adulta mayor en condición de abandono o de vulnerabilidad social. La capacidad del espacio se destina a 39 personas adultas mayores. La infraestructura incluye cuartos individuales con espacios para cuidado personal, baños compartidos, salones compartidos tipo sala de estar, un comedor y un salón grande para actividades recreativas y de ocio.

En el marco del presente estudio, se aplicó el instrumento a un total de 23 personas adultas mayores, debido a que únicamente estas cumplían con los criterios de inclusión de la población participante. A continuación, se detallan los resultados: 
Figura 1. Distribución de personas adultas mayores entrevistadas en el Hogar de Ancianos de Coto Brus por sexo y rango de edad, para el mes de noviembre del 2019 (valores absolutos)

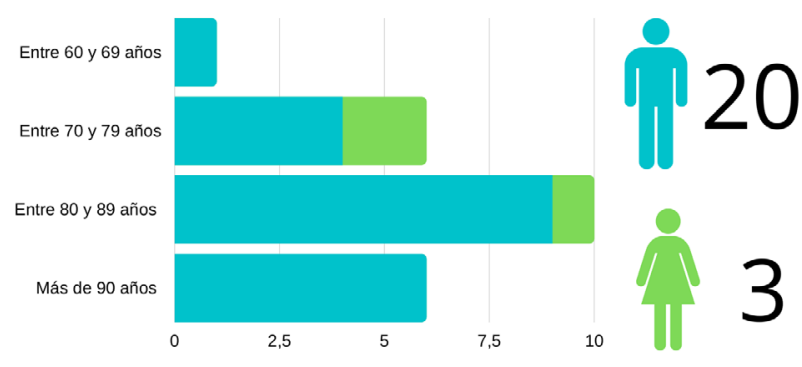

Nota: Elaboración propia, a partir de datos obtenidos, mediante el instrumento de recolección.

El $13 \%$ de la población encuestada corresponde a mujeres, el restante 87 \% corresponde a varones, diferencia proporcional entre sexo. Así mismo, como se observa en la Figura 1, la mayor cantidad de personas se ubica entre los 80 y 89 años. En la mayor edad, se encuentran 6 hombres, lo que corresponde a $30 \%$ de la totalidad de varones de la población en estudio, ellos con más de 90 años. Solo un adulto mayor se ubica entre los 60 y 69 años; es decir, la distribución real más frecuente es entre personas adultas mayores de 70 años.

Otro aspecto, sobre el cual se consultó, fue acerca de la creencia religiosa de las personas participantes en el estudio. En la Figura 2 se destaca que la mayoría se identifica dentro una denominación religiosa basada en el cristianismo. 
Figura 2. Creencia religiosa de las personas adultas mayores del Hogar de Ancianos de Coto Brus por sexo, para el mes de noviembre del 2019 (valores absolutos)

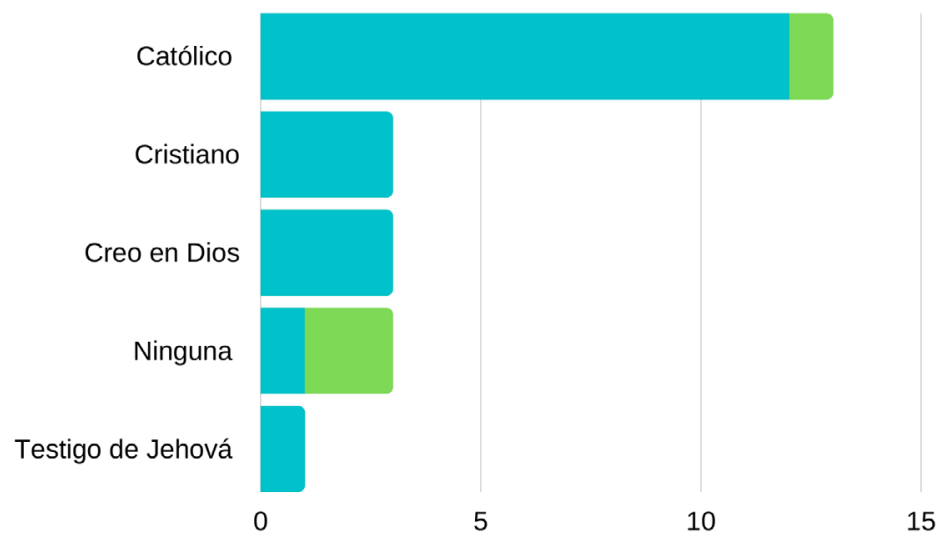

Nota: Elaboración propia, a partir de datos obtenidos, mediante el instrumento de recolección.

En relación con la creencia religiosa, únicamente tres, dos son mujeres y un varón, refieren no tener ninguna creencia religiosa, lo que corresponde a un $13 \%$ del total de la población en estudio. Mientras tanto, el restante 87 $\%$ manifiesta alguna creencia. En la Tabla 2 se muestra dicha distribución. Vale la pena mencionar que la aceptación o el reconocimiento de una religión cristiana implica, necesariamente, la apropiación de una concepción de la muerte basada en lo que estas religiones profesan. Por tanto, en muchos sentidos, la forma en cómo se enfrenta este proceso de preparación para el momento de morir estará teñida, e incluso, basada en lo aprehendido mediante la relación con el cristianismo.

Dentro de este proceso de preparación para la muerte, hay quienes visualizan detalles del momento y de las circunstancias de este evento concluyente de la vida. Otras personas no piensan en ello, o quizá nunca lo hayan determinado. En este sentido, fue de interés en este estudio consultar por el lugar en el cual las personas participantes preferirían morir. 
Figura 3. Distribución porcentual del lugar donde preferiría morir la persona adulta mayor encuestada en el Hogar de Ancianos de Coto Brus, para el mes de noviembre del 2019

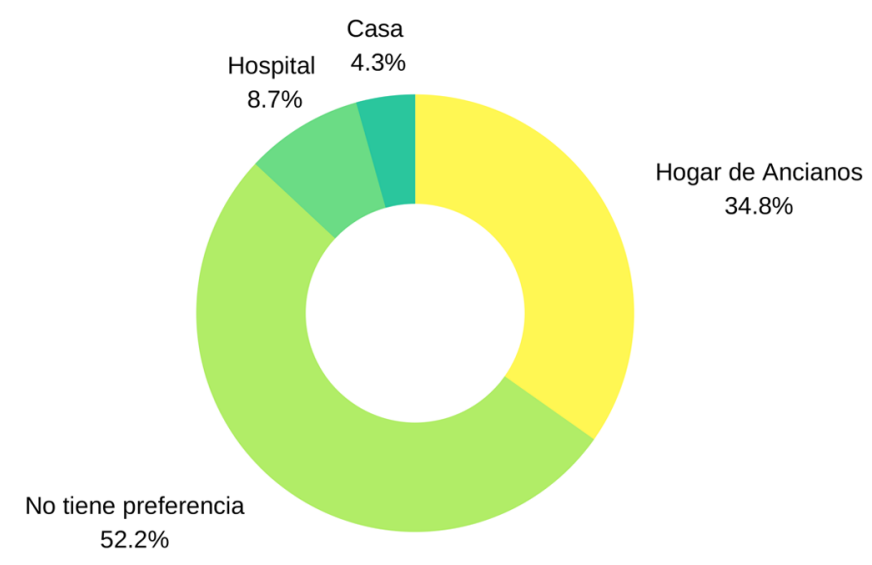

Nota: Elaboración propia, a partir de datos obtenidos, mediante el instrumento de recolección.

De acuerdo con la Figura 3, la mayoría de las personas consultadas no expresa preferencia con respecto del lugar destinado a morir (52\%), mientras que un $4,3 \%$, el menor porcentaje, elegiría morir en su casa. Un $8,7 \%$, en el hospital y un 34,8 \%, en el Hogar de Ancianos.

Otro aspecto, que se consideró en la consulta, fue acerca del acompañamiento en los últimos momentos de vida. Resultó esencial conocer, si existía alguna preferencia en relación con personas cercanas u otros actores dentro del proceso. 
Figura 4. Acompañante indicado por la persona adulta mayor del Hogar de Ancianos de Coto Brus, que le gustaría que estuviera presente en el momento de su muerte, para el mes de noviembre del 2019 (valores porcentuales)

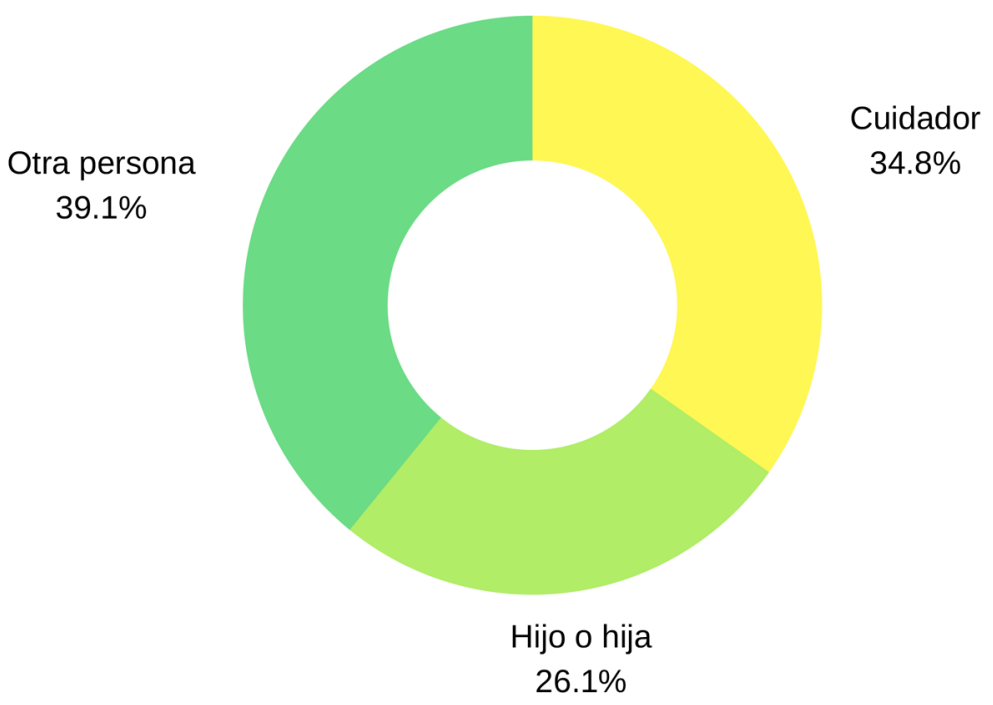

Nota: Elaboración propia, a partir de datos obtenidos, mediante el instrumento de recolección.

Al consultar sobre quién sería la persona que les gustaría le acompañe en el momento de su muerte, se destaca con mayor porcentaje $(39,1 \%)$ otra persona, seguido de los cuidadores o las cuidadoras: en algunos casos, refieren, por ejemplo, que sea alguien del mismo Hogar de Ancianos; en otros, cualquier otra persona cuidadora.

Dentro del estudio, además de situaciones de preferencias hacia sus vínculos afectivos, existe una serie de consultas sobre decisiones anticipadas en relación con el momento de morir y la asistencia que pudieran tener o no en este proceso. Para ello, se consultó sobre alimentación artificial, maniobras de resucitación, medidas para alargar la vida y posibilidad de donación de órganos. Las respuestas recibidas, en este sentido, se organizan de la siguiente manera: 
Figura 5. Distribución de porcentaje de disposición a que se le realice maniobras de resucitación o que se le aplique medidas para alargar la vida en las personas adultas mayores del Hogar de Ancianos de Coto Brus, por sexo, para el mes de noviembre del 2019

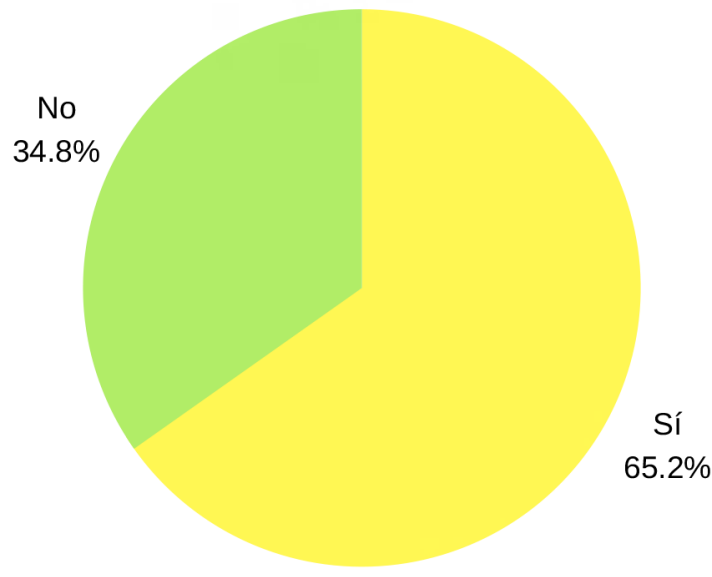

Nota: Elaboración propia, a partir de datos obtenidos, mediante el instrumento de recolección

Aun cuando, en el cuestionario, estos elementos (maniobras de resucitación y medidas para alargar la vida) se indagaron de manera separada, en la Figura 5 se presentan en conjunto, porque las respuestas fueron exactamente las mismas. De esta manera, se puede reconocer, claramente, que la intención de mantenerse con vida pesó en un $65,2 \%$ sobre el hecho de no recibir asistencia para extender la vida.

Sin embargo, una contraposición interesante identificada corresponde a que, al consultar sobre la posibilidad de recibir alimentación artificial, la posición cambia, tal como se observa a continuación: 
Figura 6. Distribución porcentual de cantidad de personas adultas mayores en el Hogar de Ancianos de Coto Brus, que estarían dispuestas a permitir o aceptar que se le brinde alimentación artificial, para el mes de noviembre del 2019

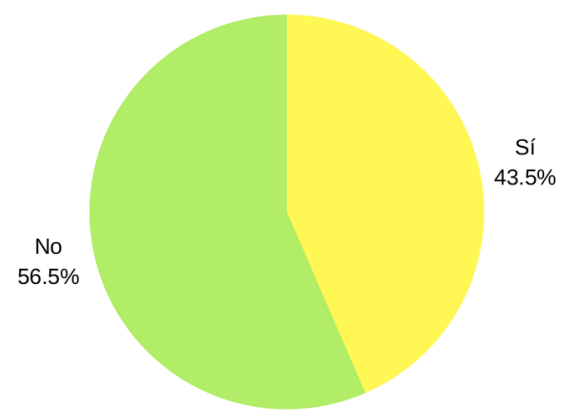

Nota: Elaboración propia, a partir de datos obtenidos, mediante el instrumento de recolección.

La mayoría de las respuestas $(56,5 \%)$ se inclinó hacia no permitir o aceptar alimentación artificial para mantenerse con vida. Aunado a ello, la posibilidad de donar órganos, al ser este un tema en la zona tan poco conocido fue interesante identificar lo que se muestra en la Figura 7.

Figura 7. Distribución porcentual de personas adultas mayores en el Hogar de Ancianos de Coto Brus, con disposición de donar sus órganos, para el mes de noviembre del 2019

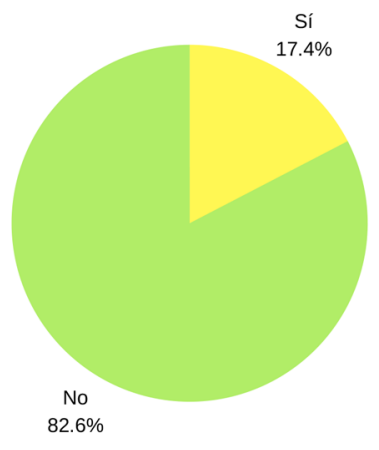

Nota: Elaboración propia, a partir de datos obtenidos, mediante el instrumento de recolección. 
Un 17,4 \% de las personas adultas mayores encuestadas estaría dispuesta a donar sus órganos en caso de requerirse. También, muchas de las respuestas giraban alrededor de que no quisieran que otras personas sufrieran sus malestares o, en su defecto, el hecho de no estar seguros de si los órganos están en buen estado. Un aspecto interesante identificado en las respuestas fue que la intención, de donar o no, podría cambiar, si el caso de la persona que requiriera el órgano fuera familiar directo. Sin embargo, al consultar, si ellos autorizarían la donación de órganos de familiares, en caso de muerte, indican que no lo autorizarían, si de ellos o ellas dependiera la decisión.

Para finalizar, dentro de la posición sobre qué hacer con los restos, una vez que se diera el fallecimiento, las respuestas se presentaron de manera muy clara y con una posición fuerte hacia la posibilidad de ser enterrados, tal como se observa en el gráfico siguiente:

Figura 8. Distribución porcentual de la preferencia sobre disposición de los restos, una vez haya fallecido la persona adulta mayor en el Hogar de Ancianos de Coto Brus, para el mes de noviembre del 2019

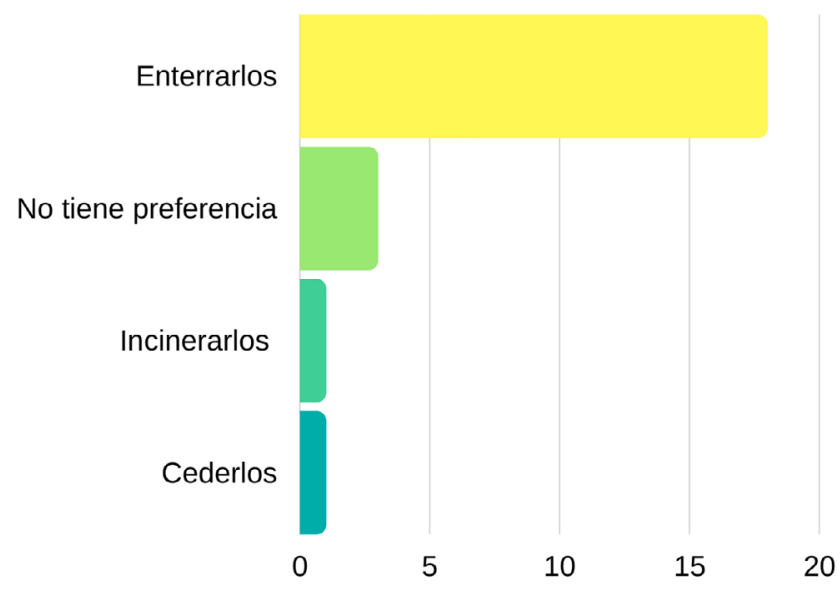

Nota: Elaboración propia, a partir de datos obtenidos, mediante el instrumento de recolección.

Así, se evidencia que los métodos tradicionales persisten en la intención de disposición de restos de las personas adultas mayores. Llamó la atención que uno de ellos mencionó: "quisiera que me entierren como antes, en la 
pura tierra, sin más en un hueco y listo". Con ello, se ratifican las tradiciones y lo aprendido desde tiempos anteriores.

Otro aspecto por destacar es el hecho de que $13 \%$ no señala preferencia alguna sobre el espacio. Por ejemplo, refieren que una vez fallecidos les resulta indiferente lo que hagan con el cuerpo. En algunos casos, se evidenció una necesidad económica. Hubo una respuesta mediante la que se indicó: "que hagan lo que les salga más barato, o lo que puedan hacer desde las posibilidades de a quién le corresponda, hacerse cargo de mis restos".

\section{Discusión de resultados}

En nuestra cultura occidental tratar temas relacionados con la vejez, la enfermedad y la muerte suele generar resistencia. Quizá, dicha indisposición se relacione con que se trata de tópicos que confrontan a la persona con su propia vulnerabilidad y le obligan a tomar decisiones difíciles que, muchas veces, implican pérdidas y sufrimiento. En estas circunstancias, tomar decisiones puede no ser tarea sencilla, porque se realiza en medio de una emocionalidad adversa y de un sentido de carencia, que podría resultar abrumador e incluso desesperanzador, debido a que se vincula con diferentes elementos de la vida de alta relevancia para la persona.

Ya es reconocido que un proceso complejo en la vida de las personas es, precisamente, aquel en el cual se toman decisiones. Todo ello porque la situación por sí misma enfrenta al ser humano con la incertidumbre del efecto de la decisión, no solo para sí mismo, sino también para los otros. La toma de decisiones suele ser un aspecto mental de la persona, pero se ve influenciada por diversos factores como la familia, las creencias, conocimientos, interpretaciones, sensaciones y percepciones de la sociedad (Ibrahimova, 2015).

En muchos casos, se deben tomar decisiones con antelación que son relevantes para pasar los últimos momentos de vida, o bien, antes de morir. Por ejemplo, el decidir con quién se desea pasar los últimos días, tal como se evidencia en las encuestas realizadas, la mayoría de residentes desea ser cuidada por otras personas o cuidadores que por miembros de su familia. Para Jiménez (2016), estas decisiones se tornan relevantes

La valoración de las decisiones autónomas, desde el respeto y la consideración, requiere tomar en cuenta todo aquello que pueda afectar a la persona y su mundo valorativo. La desestimación, el ignorar o insultar las voluntades 
de otros, es una acción que irrespeta la autonomía y el proyecto de vida de los demás. (p. 98)

Para el momento de la entrevista, las personas cercanas de la población en estudio son aquellas con las que se relacionan en el presente, sus nuevos cuidadores, las personas con quienes intercambian día tras día. Las personas con las que se relacionan en el Hogar, muy posiblemente, son quienes les conocen en sus nuevas necesidades y vulnerabilidades. No es extraño que resulten ser sus personas de confianza y con las cuales se sienten seguros para enfrentar los últimos momentos de sus vidas.

Los familiares o los amigos del pasado, a pesar de que pueden sostener con ellos lazos afectivos y parentales, ya no son de su círculo directo, ni les ubican, necesariamente, como capaces de cuidarles o asegurarles que saben sobre sus necesidades de salud. Además, se suma que, independientemente del tiempo que lleven dentro del Hogar, sus familiares o convivientes del pasado, habrán variado sus dinámicas de vida y, con ello, se instaura el sentido de no pertenencia a ese espacio evolucionado en el tiempo, que ya no les es propio, ni ellos forman parte de ese nuevo sitio.

De lo anterior, puede no resultar extraño, entonces, que se perciba una especie de desarraigo con la familia, mediante el cual no se elige la casa familiar como la primera instancia para esperar la muerte, ni tampoco se visualiza necesariamente a los hijos e hijas como las personas más idóneas para acompañar en el proceso hacia la muerte.

La OPS (2004), muy relacionado con los anteriores planteamientos, menciona:

El envejecimiento poblacional tiene y tendrá un impacto importante sobre la estructura familiar. Por ejemplo, con la edad aumenta también la probabilidad de que los adultos mayores dependan de otras personas, especialmente aquellos que forman parte de un mismo núcleo familiar. (OPS, 2004, p. 11)

Queda preguntarse si el concepto de núcleo familiar, en condiciones de institucionalización, para personas adultas mayores, se debe mantener tal cual, en el tiempo y en las diferentes circunstancias, porque, a todas luces, para la persona adulta mayor en institución, su círculo cercano habrá cambiado y, con ello, puede que el concepto de núcleo familiar debería ser revisado. 
Se puede reconocer en los planteamientos anteriores el papel preponderante que cumplen, en la sociedad, los hogares para personas adultas mayores, puesto que no solo significan espacios que ofrecen condiciones dignas a personas que requieren apoyo en esa etapa de la vida, sino también restablecen, en la persona, la existencia de un nuevo círculo cercano, que le devuelve el sentido de pertenencia.

Si a lo anterior se suma que el envejecimiento de la población está ocurriendo aceleradamente en Costa Rica, en un periodo mucho más corto que en los países más desarrollados, Los 301.000 adultos mayores contabilizados en el censo de 2000 pasarán a ser cerca de 2 dos millones en el 2060 (OPS, 2004, p. 48).

Sin duda, esto apunta a que será cada vez más imperante y necesario generar recursos de apoyo para enfrentar los procesos propios del proceso de vejez, enfermedad y muerte. "A pesar de que son pocos los adultos mayores que viven en instituciones, el porcentaje aumenta conforme aumenta su edad y por tanto su dependencia funcional (Organización Panamericana de la Salud" (OPS, 2004, p. 14).

Un dato particularmente interesante, que se desprende de la indagatoria realizada, es la significativa mayor institucionalización de hombres que de mujeres. No es un dato aislado y se puede reconocer en otros estudios.

Sin embargo, en la mayoría de grupos de edad es mayor el porcentaje de hombres en instituciones. Esto probablemente se debe a que el vínculo filial es más débil con los padres que con las madres, lo cual puede deberse a que los hombres hayan abandonado en el pasado sus obligaciones paternas, lo que amerita estudios para profundizar en el análisis que explique esta situación. (OPS, 2004, p. 15)

El planteamiento de la OPS es, sin duda, válido y probable en muchos casos; sin embargo, pueden suponerse otras razones por las cuales se da este fenómeno de la mayor institucionalización masculina. Una razón puede ser el juego del rol femenino en las dinámicas familiares al ser que la mujer adulta mayor puede valorarse más en los entornos de familia por sus capacidades de aportar al mantenimiento de las casas, cuido de infantes, preparación de alimentos, entre otras labores tradicionalmente asignadas a las mujeres y, por tanto, más probablemente dominadas por este género. 
Un dato que llama la atención en los resultados de la encuesta es que, proporcionalmente, son más las mujeres las que manifiestan no tener una creencia religiosa. Nótese que se consultó sobre la afiliación a una organización religiosa y no necesariamente a la condición de ser creyentes o no. Además, debe tomarse en cuenta que la cuota de participación de mujeres, en términos absolutos, es muy baja y ello dificulta llegar a planteamientos más definitivos. Sin embargo, sí se podría especular en aspectos tales como que su condición de persona institucionalizada puede haber significado un corte con respecto a sus costumbres y participaciones en este tipo de organizaciones. Más allá de los supuestos, es muy rescatable reconocer que se vive en una época en la cual mujeres adultas mayores se sienten y están en la libertad de declararse como no practicantes de religión alguna. Ello refleja, sin duda, un avance hacia una mayor tolerancia de las diversidades de opinión y estilos de vida.

Mucho, en la línea de la situación global de Costa Rica mencionada por Fuentes (2016), expone que, durante tres siglos, la Iglesia Católica garantiza la administración del territorio costarricense, así como la educación de sus residentes y el adiestramiento de sus cuerpos. Si bien después de la independencia de la República, la Iglesia se aclimata a los cambios sociales notoriamente mediante el mantenimiento de la afinidad electiva con las élites políticas, esta no pierde su influencia sobre el imaginario nacional, que se gesta en la construcción del Estado-Nación, a pesar de los brotes del movimiento neopentecostal, creencias no cristianas, o el aumento de quienes no profesan ninguna religión.

Sin embargo, los resultados del estudio realizado por Fuentes (2016) se contraponen a lo identificado con el comportamiento del grupo en estudio, debido a que, según ella, en referencia al género, las mujeres tienden a identificarse más con prácticas religiosas y sus posiciones tienden a ser más conservadoras en cuanto a política, moral y sexualidad, que las de los hombres. No obstante, la realidad de la población de estudio del Hogar de Ancianos de Coto Brus presenta que las mujeres, en su mayoría, no se identifican con alguna práctica religiosa.

La mayoría de las personas consultadas indica que aceptaría recibir reanimación y la aplicación de métodos para alargar la vida. Sin embargo, al considerar recibir alimentación artificial la balanza cambia. Nótese que, 
alargar la vida, no lleva explícita la presencia de la enfermedad. Más bien, se plantea en términos favorables y positivos. La posibilidad, en sí misma, apunta a una adición a la vida. Sin embargo, en el caso de recibir alimentación artificial, necesariamente refiere a una condición de deterioro de la salud, a la enfermedad y al dolor. No implica, por tanto, una contradicción en sí misma, sino que puede estar apuntando a la visión de condiciones distintas. Al respecto, Margarita Boladeras (2013) apunta, con una precisión ética, que sitúa esta discusión en un escenario pertinente:

Asumir la enfermedad, el dolor y la muerte desde la propia vida implica poder tomar decisiones de acuerdo con la trayectoria vital de cada ser humano. El respeto a la autonomía es por lo tanto fundamental. Seguir o no un tratamiento, suspender un procedimiento, aceptar o no la hospitalización, etcétera son decisiones que dependen del enfermo y se debe velar para que así sea (pp. 226-227).

Ahora bien, queda preguntarse si, en el caso de la reflexión anterior, $\mathrm{y}$ al responder afirmativamente a la posibilidad de someterse a terapias y estrategias para alargar la vida, la persona se cuestiona sobre su estado luego de dicha decisión. Es posible que, al responder, solo visualiza la oportunidad de seguir con vida, de no morir; por lo tanto, reconoce este hecho como una ganancia.

La muerte para las personas es percibida y enfrentada de diferentes maneras, según Sarmiento et al. (2012, citado por Ibrahimova, 2015, p. 15):

Aunque para cada paciente son diferentes, todos coinciden en que se debe morir con el mínimo dolor posible, sin sensación de asfixia, sin intervenciones tecnológicas invasivas, sin una prolongación de la vida cuando no hay pronósticos de mejoría, rodeado de afecto, en un contexto de respeto por sus costumbres, creencias y voluntades, y preferiblemente en casa.

Son estas las reflexiones que quizá se dejan de lado cuando se valora la posibilidad de alargar la vida sin entrar en una reflexión más detallada y profunda de lo que ello implica, de qué puede venir después.

La mayoría de las personas adultas mayores consultada (casi $80 \%$ ) indicó que, para efectos de la disposición de sus restos, una vez acaecida la 
muerte, de preferencia, quisieran ser enterrada. Esto es coincidente con el planteamiento de Philippe Aries (2000), quien plantea que en este tema hay una mayor inclinación a elegir métodos tradicionales para el tratamiento de los cuerpos fallecidos (tradición). Contar con este dato será importante para los familiares y el Hogar de Ancianos, porque ofrece una certeza sobre el deseo de sus residentes.

En los resultados de las encuestas se evidencia que las decisiones relevantes, como el donar los órganos, no son influenciadas directamente por las creencias religiosas (católico, testigo de Jehová, creer en Dios, cristiano o no tener ninguna creencia religiosa) por lo que cada persona es influenciada por sus valores individuales que, en muchas veces, ocasionan dilemas éticos (Ibrahimova, 2015). Vale la pena, en este caso, preguntarse sobre la razón por la cual se da esta resistencia a la donación. Quizá, deba indagarse sobre los motivos que fundamentan esta negativa. Ello podría aportar recursos para que el Hogar tome acciones de información u otras estrategias, que pudieran ser útiles a las personas adultas mayores con respecto a asumir una decisión sobre este tema.

En este estudio, el $52 \%$ de las personas encuestadas manifestó no tener preferencia con respecto a dónde le gustaría morir, seguido por la elección de morir en un hogar de ancianos (34.8 \%) y con menor frecuencia de respuesta se mencionó el hospital y la casa de familia. Esta última contó con el menor número de respuestas. Pareciera que el evento de la muerte no necesariamente se piensa como una situación para experimentarla en un sitio en particular. Las respuestas, quizá, tienen de fondo el sentir de que la muerte llegará como desenlace irremediable sin distinción del lugar en el cual se encuentre la persona, sin asociar una mejor o peor situación en dependencia del lugar donde acaece este evento. Además, con respecto al alto porcentaje que indica no tener preferencia, podría preguntarse si efectivamente no se cuenta con una inclinación hacia algún lugar para ese momento o es un tema sobre el cual no se ha pensado.

Uno de los aspectos más sobresalientes de este estudio es el reconocimiento de que, hacia el final de la vida, también, se deben tomar decisiones de gran trascendencia para las personas. Son muchos los elementos sobre los cuales se debe reflexionar y varias las acciones que median para llevar a la práctica las decisiones que tomen las personas. Significa, sin duda, un 
derecho que debe ser garantizado y el sistema debe contribuir a que se cuente con las condiciones para respetar dichas decisiones.

\section{Conclusiones}

Las personas requieren expresar sus expectativas y sus opiniones en relación con temas referentes a su autonomía. La consulta sobre los deseos, las expectativas, las intenciones y las voluntades en el proceso del final de la vida se transforman en cruciales para cada sujeto. Por ello, es relevante trabajar de la mano de las personas usuarias de los centros y hogares de cuido para el abordaje de este proceso. La consigna debe ubicarse en favorecer el diálogo para establecer mecanismos que privilegien la seguridad y la confianza en que lo que cada sujeto indique será considerado cuando se aproxime o llegue el momento decisivo.

El lugar donde morir constituye uno de los aspectos que genera, en no pocas veces, situaciones de angustia. Sin embargo, para la muestra de este estudio, más del $50 \%$ manifestó no tener preferencia. En este sentido, es relevante que casi un $34 \%$, indique que preferirían el Hogar de Ancianos. Asimismo, un dato interesante consiste en la ausencia de la familia o casa de un familiar como el ambiente para morir. Es necesario preguntarse, si esta inclinación en la respuesta evidencia que, efectivamente, no hay una preferencia sobre el lugar en el que desean morir, o de fondo se debe a que no se ha reflexionado al respecto del tema, en cuyo caso sería recomendable abrir espacios para dialogar sobre este asunto.

Un rasgo que requiere ser ampliado y mayormente estudiado es la relación con la familia. Lo anterior, por cuanto, la preferencia por una persona que cuide en el proceso del final de la vida no pertenece al núcleo familiar, sino a categorías como otra persona o cuidador. Las razones del desarraigo requieren ser indagadas, porque podrían ser fuente para la toma de decisiones, que afecten la disposición de la persona adulta mayor en lo relativo a las voluntades en este proceso.

El dato de la negativa para donar los órganos requiere ser considerada y abordada en futuros estudios. Esto, por cuanto es una de las decisiones más complejas que puede considerar un sujeto, debido, en no pocos casos, a creencias religiosas. Por ello, resulta oportuno indagar esta decisión para nuevos estudios. 
La necesidad de considerar la implementación de consultas sobre las voluntades ante el proceso del final de la vida forma parte de los servicios que se ofrecen en el hogar de ancianos. En el pasado se consideró que todos los sujetos contaban con una identidad relativamente uniformada y que ello implicaba consideraciones morales similares en temas de final de la vida. Al respecto, es oportuno pasar a una práctica de consulta constante y preferiblemente mediante la adopción de un protocolo, donde se consignen las voluntades de los sujetos morales ante el proceso del final de la vida.

\section{Referencias}

Alcohoberro, R. (2011). La autonomía la final de la vida. En M. Boladeras (Ed.), Bioética: La toma de decisiones. Proteus.

Asociación Hogar de Ancianos de Coto Brus. (1989). Carta Constitutiva. San Vito, Coto Brus, Puntarenas.

Beauchamp, T. y Childress, J. (2013). Principles of Biomedical Ethics ( $7^{\mathrm{ma}}$ ed.). Oxford University Press.

Boladeras, M. (2013). El impacto de la tecnociencia en el mundo humano: Diálogos sobre bioética. Tecnos.

Cabrera, M. y Sales, P. (2013). Vulnerabilidad en los cuidados paliativos. En M. Boladeras, Bioética: Justicia y vulnerabilidad. Proteus.

Fuentes Belgrave, L. (2016). Cambios en las creencias religiosas en Costa Rica. SIWÖ, 9,(1). Universidad Nacional de Costa Rica. http://dx.doi.org/10.15359/siwo.9-1.3

Ibrahimova, A. (2015). Proceso de toma de decisiones al final de la vida [Tesis de grado]. Universidad Pública de Navarra. España. https://academica-e.unavarra. es/bitstream/handle/2454/18699/Atidzhe\%20Ibrahimova\%20Bashchobanova. pdf? sequence $=1$ \&isAllowed $=\mathrm{y}$

Instituto Nacional de Fomento Cooperativo. (2005). Vejez, dignidad y productividad: Un estudio exploratorio de las condiciones de los y las adultas mayores en Costa Rica. https://www.infocoop.go.cr/sites/default/files/2020-01/8.\%20Vejex\%2C\%20dignidad $\% 20 y \% 20$ productividad.pdf

Jiménez, R. (2016). Voluntades anticipadas: Derecho a decidir. Revista Praxis, 74, 95-108. https://doi.org/10.15359/praxis.74.5

Jiménez, R. (2018). Voluntades anticipadas: Caso Costa Rica. En A. Richart, M. García-Granero, C. Ortega Esquembre y L. de Tienda Palop (Eds.). Juicio moral y democracia. Retos de la ética y la filosofía política. Comares.

Kubler-Ross, E. (1993). Sobre la muerte y los moribundos. Debolsillo.

Organización Panamericana de la Salud. (2004). Situación de la persona adulta mayor en Costa Rica. San José Costa Rica.

Tolstói, L. (2002). La muerte de Ivan Illich. Escolares. 
